\title{
Ubiquitous yet Distinct Expression of Podocalyxin on Vascular Surfaces in Normal and Tumor Tissues in the Rat
}

\author{
Jacqueline E. Testa Adrian Chrastina Yan Li Phil Oh Jan E. Schnitzer \\ The Sidney Kimmel Cancer Center, San Diego, Calif., USA
}

\section{Key Words}

Caveolae $\cdot$ Immunofluorescence $\cdot$ Immunohistochemistry • Molecular imaging $\cdot$ Biodistribution $\cdot$ SPECT imaging

\begin{abstract}
Background/Aims: The vasculature has become an important target in the development of therapies for increasing numbers of human diseases, yet there are few reliable markers available to identify the endothelium in rodent models. We have characterized the expression, subcellular localization and accessibility of the rat pan-endothelial marker podocalyxin (podxl) using a newly developed monoclonal antibody (mAb), G278. Methods: podxl expression and accessibility to binding by $\mathrm{G} 278$ were determined in the rat by a variety of experimental approaches. Results: mAb G278 reliably immunostained blood vessels of all types and of every size in fresh-frozen, fixed-frozen and paraffin-embedded sections of all tissues, but did not stain lymphatic vessels. Western blotting, in vivo imaging and biodistribution analyses demonstrated that the highest levels of endothelial podxl were found in the lung and heart. We also determined that podxl is not enriched in caveolae and that its expression can be modulated in the tumor microenvironment. Conclusion: Our study shows that podxl is a better identifier of rat endothelia than are some of the more commonly used markers and that mAb G278 is a robust antibody for use not only in identifying rat blood vessels but also as a tool to elucidate podxl function.

Copyright $\odot 2009$ S. Karger AG, Basel
\end{abstract}

\section{Introduction}

The endothelium is the interface which separates circulating blood from underlying tissues and mediates the exchange of nutrients, metabolic waste products and other molecules to maintain normal physiological function [1]. In addition to its role in normal homeostasis, the vasculature is associated with an array of pathological conditions brought on by or supported by either excessive angiogenesis (e.g. cancer, diabetic retinopathy, age-related macular degeneration, rheumatoid arthritis) or insufficient angiogenesis (e.g. ischemic heart disease, delayed wound healing, osteoporosis; for a review see [2]). Because of the strong relationship between disease and imbalances in angiogenesis, the vasculature has become an important therapeutic target for an increasing number of diseases. Developing molecules that can effectively target blood vessels relies heavily on the use of animal models and therefore requires reagents such as monoclonal antibodies (mAbs) that can reliably and accurately identify the vasculature in a variety of experimental methods. Unfortunately, there are few such antibodies, especially those that can identify the rat endothelium.

The focus of our research is to map the rat endothelial cell surface proteome and to identify accessible vascular targets using a variety of techniques including proteomics analysis based on mass spectrometry, and hybridoma and phage technologies [3-6]. During the course of this work, we have produced a number of antibodies to selected tissue and tumor-induced protein targets. Here we utilize

Dr. Jan E. Schnitzer and Dr. Jacqueline E. Testa

The Sidney Kimmel Cancer Center

10905 Road to the Cure, San Diego, CA 92121 (USA)

Tel. +1 858450 5990, ext. 327, Fax +1 8584503251

E-Mail karger@karger.ch Accessible online at:

www.karger.com www.karger.com/jvr

E-Mail jschnitzer@skcc.org, jtesta@skcc.org 
one of these antibodies, mAb G278, to characterize and evaluate the expression and distribution of the pan-endothelial marker podocalyxin (podxl) in normal and tumor tissues in the rat.

\section{Methods}

Animals

All animal experiments were carried out in accordance with protocols approved by the Institutional Animal Care and Use Committee of the Sidney Kimmel Cancer Center (SKCC). Animals were housed in the SKCC animal care facility, and those animals which received radiolabeled antibodies were housed and imaged in a separate lead-shielded animal facility at SKCC. Fischer rats (150$250 \mathrm{~g}$ ) were obtained from Charles River Laboratories, and BALB/ C mice (6-8 weeks) were purchased from Jackson Laboratories.

\section{Rat Tumor Models}

The rat sarcoma cell line FRcJ-4 was a kind gift from Dr. Bernard Binétruy (Director of Research, INSERM, University of Nice, France). Lung tumors were initiated by injecting rats via the tail vein with FRcJ-4 cells or the breast carcinoma cell line 13762 as previously described $[7,8]$. Tumor-bearing lungs were prepared for sectioning as described below.

\section{Hybridomas, Monoclonal and Polyclonal Antibodies}

$\mathrm{BALB} / \mathrm{C}$ mice were immunized with luminal endothelial cell plasma membranes $(\mathrm{P})$ isolated from rat lungs using the silica coating technique [7-11]. Each mouse was injected subcutaneously with $300 \mu \mathrm{g}$ of P suspended in Freund's complete adjuvant, followed by two boosts with $300 \mu \mathrm{g}$ of P in Freund's incomplete adjuvant. Serum was collected from tail bleeds taken after the first and second boosts, diluted in block (5\% FBS in PBS) by serial doubling dilutions and the titers were measured by P ELISA (see below). The mouse with the highest titer after the second boost was given a final injection of $300 \mu \mathrm{g}$ of soluble P (i.e. no Freund's) and the spleen was harvested 5 days later. Splenocytes were fused with P3X.63-Ag8.653 myelomas using polyethylene glycol and selected in hypoxanthine aminopterin thymidine medium following standard protocols [12]. At 10 and 12 days after the fusion, half of the medium (i.e. $100 \mu \mathrm{l}$ ) was removed from the wells in the fusion plates and replaced with fresh hypoxanthine aminopterin thymidine medium. The fusion plates were then screened by P ELISA on the 14th day after fusion to identify wells containing hybridomas producing antibodies against rat endothelial cell antigens. Selected parent hybridomas were expanded in culture and the culture supernatants were tested on Western blots of rat lung homogenate and rat lung P. Parent hybridomas producing antibodies that recognized an antigen which was significantly enriched in $\mathrm{P}$ were subjected to two rounds of subcloning to ensure clonality.

Rabbit anti-rat podoplanin was purchased from Sigma-Aldrich (St. Louis, Mo., USA). Goat polyclonal antibody (pAb) to rat PECAM/CD31 (M20) was purchased from Santa Cruz Biotechnologies Inc. (Santa Cruz, Calif., USA).

Antibody Purification and Isotyping

Clarified hybridoma culture supernatants were passed over a bed of GammaBind Plus (GE Healthcare, Piscataway, N.J., USA) and after washing with 10 bed volumes of PBS, the antibody was eluted with glycine $(0.1 \mathrm{M}, \mathrm{pH} 3.0)$ into tubes containing $25 \mu \mathrm{l}$ HEPES (1.0 M, pH 7.0). Peak fractions were collected and dialyzed against $\mathrm{PBS}$ at $4^{\circ} \mathrm{C}$ overnight with stirring. Sodium azide was added and the antibody was stored at $4^{\circ} \mathrm{C}$. Isotyping was done with the Clonotyping System-HRP from Southern Biotechnology following standard protocols.

\section{P ELISA}

Rat lung $\mathrm{P}$ was diluted to a concentration of $1.0 \mathrm{mg} / \mathrm{ml}$ in water and sonicated on ice. The sonicated $\mathrm{P}$ was diluted to a final concentration of $0.1 \mathrm{mg} / \mathrm{ml}$ in water and pipetted into the wells of a 96 -well plate at $50 \mu \mathrm{l} /$ well. The plates were placed, uncovered, in a $37^{\circ} \mathrm{C}$ oven and allowed to dry overnight. Prior to use, the plates were blocked with 5\% FBS in PBS for $1 \mathrm{~h}$. The block was removed and primary antibody (i.e. diluted serum from tail bleeds or $50 \mu \mathrm{l}$ of medium from fusion plates) was added and incubated for $2 \mathrm{~h}$ at room temperature. The plates were then washed 3 times with PBS and horseradish-peroxidase-conjugated goat antimouse immunoglobulin (Ig) secondary antibody (KPL, Gaithersburg, Md., USA), diluted in block, was added and incubated for $1 \mathrm{~h}$ at room temperature. The secondary antibody was removed by washing three times with PBS and $75 \mu \mathrm{l}$ of the substrate $o$ phenylenediamine (Sigma-Aldrich) was added to the wells. The reaction was stopped after $5 \mathrm{~min}$ by adding an equal volume of $2 \mathrm{~N} \mathrm{H}_{2} \mathrm{SO}_{4}$ and the plates were read at $490 \mathrm{~nm}$.

\section{Western Blotting}

Samples $(5 \mu \mathrm{g})$ of rat organ homogenates and $\mathrm{P}$ were boiled in $1 \times$ reducing sample buffer and proteins were resolved by sodium dodecyl sulfate polyacrylamide gel electrophoresis (SDS-PAGE) and then transferred to nitrocellulose. The equality of the protein loads was verified by staining the nitrocellulose blot with Ponceau $\mathrm{S}$ and/or by silver staining a duplicate PAGE gel. Blots were used only if the variation between samples was less than $10 \%$. Blots were blocked for $30 \mathrm{~min}$ at room temperature in $2 \%$ powdered milk dissolved in PBS/0.1\% Tween 20 followed by a 2-hour incubation with the primary antibody (hybridoma culture medium diluted 1:10 in PBS $/ 0.1 \%$ Tween 20 , or purified antibody diluted to $2 \mu \mathrm{g} / \mathrm{ml}$ in block). The blots were then washed and incubated in horseradishperoxidase-conjugated goat anti-mouse Ig (GE Healthcare) for $1 \mathrm{~h}$ at room temperature. After additional washes, signals were visualized by saturating the blots with SuperSignal West Pico (Pierce, Rockford, Ill., USA) and exposing them to X-ray film.

\section{Tissue Preparation and Immunostaining}

To prepare tissues for paraffin embedding, rats were anesthetized and the vasculature was perfused with Ringer's followed by $10 \%$ neutral buffered formalin (NBF). The tissues were removed, cut into $0.5-\mathrm{cm}$ slices and postfixed for $6 \mathrm{~h}$ in NBF prior to processing and embedding in paraffin. Sections $(5 \mu \mathrm{m})$ were mounted on Colorfrost/Plus slides (Fisher Scientific, Hampton, N.H., USA). Tissue for cryosections were also fixed by perfusion with NBF and then perfused with $30 \%$ sucrose in NBF. The tissues were removed, cut into $0.5-\mathrm{cm}$ slices, postfixed for $6 \mathrm{~h}$ in $30 \%$ sucrose in NBF and then placed in $30 \%$ sucrose in PBS overnight at $4^{\circ} \mathrm{C}$. The tissues were embedded in TBS (Triangle Biomedical Sciences, Durham, N.C., USA) and 5 - $\mu \mathrm{m}$ sections were cut on a Microm HM505E cryomicrotome and mounted on Colorfrost/Plus slides. 
Prior to immunostaining, deparaffinized, rehydrated sections were subjected to antigen retrieval in $10 \mathrm{~mm}$ citrate buffer $(\mathrm{pH}$ 6.0) in a vegetable steamer, then blocked in 5\% FBS in PBS $/ 0.1 \%$ Tween 20 at room temperature for $30 \mathrm{~min}$. Sodium azide $(0.05 \%)$ was added to the block in order to inactivate endogenous tissue peroxidases. The sections were incubated with primary antibody for $2 \mathrm{~h}$ at room temperature, washed $3 \times 5 \mathrm{~min}$ in $\mathrm{PBS} / 0.1 \%$ Tween 20 then incubated with biotinylated secondary antibody for $1 \mathrm{~h}$ at room temperature followed by horseradish-peroxidaseconjugated streptavidin (KPL). To prevent cross-reactivity with endogenous rat immunoglobulins in the tissues, a rat-serum-adsorbed, biotinylated goat anti-mouse Ig (KPL) was used to detect mouse mAbs. After washing $3 \times 5 \mathrm{~min}$, the color reaction was developed with diaminobenzidine (BioGenex, San Ramon, Calif., USA) and the sections were counterstained with hematoxylin prior to dehydration and mounting in Permount (Fisher Scientific). Digital images were acquired using a Nikon Eclipse E800 light microscope equipped with a Nikon DXM1200 digital camera.

Immunofluorescence staining of heart sections was done with paraffin-embedded tissue subjected to antigen retrieval as above. Cryosections, fixed for $5 \mathrm{~min}$ in $\mathrm{NBF}$ at room temperature, were used for lung and tumor immunofluorescence analyses. Heart, lung and tumor sections were washed in water, quenched $(1 \mathrm{mM}$ $\mathrm{CuSO}_{4}, 50 \mathrm{~mm}$ ammonium acetate in water; $20 \mathrm{~min}$ ), washed again in water then blocked and incubated with a mixture of the appropriate primary antibodies for $2 \mathrm{~h}$ at room temperature. The slides were washed $3 \times 5 \mathrm{~min}$ in $\mathrm{PBS} / 0.1 \%$ Tween 20 , then incubated for $1 \mathrm{~h}$ with Alexafluor-conjugated secondary antibodies (Invitrogen, Carlsbad, Calif., USA) in the dark at room temperature. After a final washing, sections were mounted in Vectashield (Vector Laboratories, Burlingame, Calif., USA) and confocal images were acquired with a Nikon 800 microscope fitted with a Perkin Elmer Wallac UltraView confocal head and a triple-line laser (Perkin Elmer Wallac, Gaithersburg, Md., USA).

$1 D$ Gel and LC-MS/MS Analyses

Lung $\mathrm{P}$ was incubated with $\beta$-octylglucoside (1.0\% in PBS) at $37^{\circ} \mathrm{C}$ for $1 \mathrm{~h}$ in the presence of a protease inhibitor cocktail. After centrifugation, proteins in the resulting supernatant were resolved by SDS-PAGE. Proteins were visualized by silver staining and the gel was aligned with a Western blot of the same sample probed with $\mathrm{mAb}$ G278. The appropriate gel slice was excised and proteins were digested in-gel with trypsin to yield a peptide mixture that was extracted from the gel and separated by a $\mathrm{C}_{18}$ reversed-phase microcolumn using a linear acetonitrile gradient over 60 min delivered by an Agilent 1100 HPLC system. The eluted peptides were directly introduced into a 3D ion trap mass spectrometer (LCQ DecaXP, Thermo Fisher Scientific, Inc., Waltham, Mass., USA). SEQUEST algorithms were used to designate acquired MS/MS spectra to peptide sequences from available public rat databases downloaded from UniProt and NCBI RefSeq (nonredundant) protein databases (April, 2006). Searches were performed as tryptic peptides only with a precursor mass tolerance of 2.0 Da. Accepted peptide identifications were based on a minimum $\Delta \mathrm{Cn}$ score of 0.1 and minimum cross correlation scores of $1.8(\mathrm{z}=1), 2.5(\mathrm{z}=2)$ and $3.5(\mathrm{z}=3)$. Protein identification results were extracted from Sequest outfiles and filtered and grouped with DTASelect software using the above criteria.

\section{cDNA Cloning and Recombinant Expression}

Total RNA was extracted from rat lungs with an RNeasy Kit (Qiagen, Valencia, Calif., USA) following the manufacturer's instructions. Reverse transcription (RT) was performed with $5 \mu \mathrm{g}$ of total lung RNA, 400 pmol of a podxl reverse primer 5'-TCAGAGGTGTGTATCTTCCTC-3', and 200 units of Superscript III Reverse Transcriptase (Invitrogen). Five microliters of the RT reaction was used in a polymerase chain reaction (PCR) with 100 pmol of the reverse primer and 100 pmol of forward primer $5^{\prime}$ CACCATGCGCCCCACTCTGGCG-3' modified on the $5^{\prime}$-end to facilitate cloning into the Gateway Vector system (Invitrogen). The resulting PCR product was resolved on a $0.8 \%$ agarose gel, and the single band of approximately $1,500 \mathrm{~kb}$ was excised, purified using a Perfectprep Gel Cleanup Kit (Eppendorf North America, Westbury, N.Y., USA), then cloned into the pENTR/DTOPO vector. The insert was sequenced and compared to the NCBI database and then cloned into pDEST17 using Gateway's LR reaction. Purified recombinant podxl was analyzed by mass spectrometry to verify the identity of the protein.

\section{In vivo Imaging and Biodistribution}

Purified mAb G278 and an isotype-matched control antibody were radiolabeled with ${ }^{125} \mathrm{I}$ using iodobeads (Pierce). For in vivo imaging, anesthetized rats were injected via the tail vein with radiolabeled G278 and images were captured at the time points indicated. For the biodistribution assays, $10 \mu \mathrm{g}$ of labeled G278 or the isotype-matched control IgG were inoculated via the tail vein or the left ventricle, and $1 \mathrm{~h}$ later blood samples were collected prior to transcardial perfusion with $200 \mathrm{ml}$ of PBS followed by organ dissection and measurement of bound radiolabeled antibody. Organs were also collected from animals $24 \mathrm{~h}$ after tail vein injection. Results are expressed as percent of injected dose per gram of tissue $(\% \mathrm{ID} / \mathrm{g})$.

\section{Results}

\section{ELISA and Western Blot Analysis of Proteins Expressed in Lung $P$}

A panel of $\mathrm{mAbs}$ was generated by immunizing mice with $\mathrm{P}$ following standard protocols. When the fusion was tested by lung P ELISA, a number of parent hybridomas were selected based on strong immunoreactivity to antigens expressed on rat lung endothelial cells (ECs). One of these parents, G278 (IgG1к), was selected for further study based on the ELISA results and Western blotting. In the P ELISA assay, G278 produced a very strong optical density (OD) of 2.6. By comparison, the OD from an $\mathrm{mAb}$ to aminopeptidase $\mathrm{P}$, a protein abundantly expressed in rat lung ECs $[4,13]$, was 1.2 in the same assay. When tested by Western blotting, no specific signal was observed in the lane containing total lung homogenate (fig. 1a, lane $\mathrm{H}$ ); however, because of the antigen enrichment afforded by subfractionation of the luminal plasma membrane of lung EC, a strong signal at approximately 


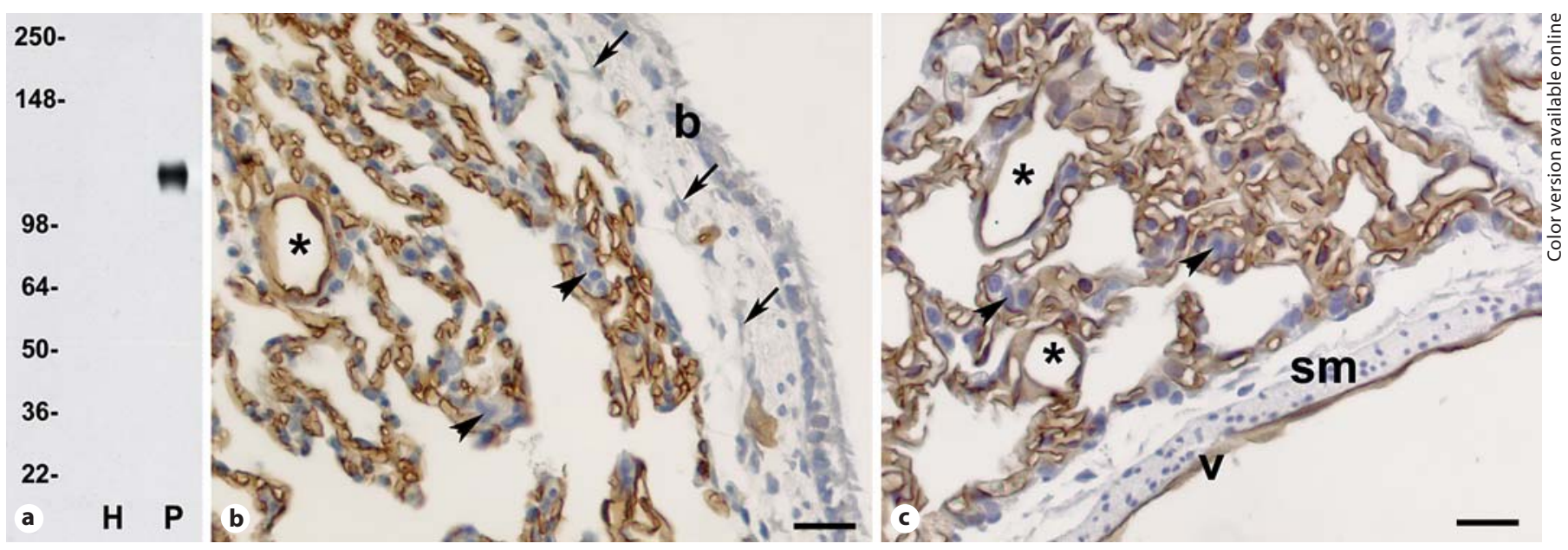

Fig. 1. a Western blot analysis of homogenate $(\mathrm{H})$ and luminal plasma membranes $(\mathrm{P})$ isolated from normal rat lungs. Five micrograms of protein were loaded in each lane and blots were probed with $\mathrm{mAb}$ G278. Molecular weight markers are on the left. b, c Immunohistochemical analysis of paraffin-embedded sections from normal rat lung. $\mathbf{b}$ Section through the wall of a bronchiole and adjoining lung parenchyma. c Longitudinal section through a large vein and the adjoining parenchyma. All ECs in all capillaries, small veins $\left(^{*}\right)$ and on the lumen of the large vein (v) are stained with G278, while fibroblasts (arrows), smooth muscle cells (sm), and epithelial cells in the alveolae (arrowheads) and bronchiole (b) are unstained. Although not shown in these images, mAb G278 also stained the endothelium of all arteries and arterioles. Bar in $\mathbf{b}, \mathbf{c}=20 \mu \mathrm{m}$.
Fig. 2. Dual immunofluorescence analysis of normal rat lung cryosections. Sections were immunostained with mAb G278 (red channel) and a goat pAb to the lymphatic marker podoplanin (green channel). There is no colocalization of the two signals, indicating that mAb G278 does not immunostain lymphatic ECs. $\mathrm{v}=$ Small vein; ${ }^{*}=$ capillary lumen. Bar $=20 \mu \mathrm{m}$.

$130 \mathrm{kDa}$ was readily detectable on blots of lung $\mathrm{P}$ (fig. 1a, lane P).

In order to verify that the signals seen on the Western blots originated from an antigen expressed on the endothelium, sections of paraffin-embedded lung were immunostained with mAb G278. A strong signal was observed on all EC while no signal was seen on epithelial cells, fibroblasts or smooth muscle cells (fig. 1b, c) or on

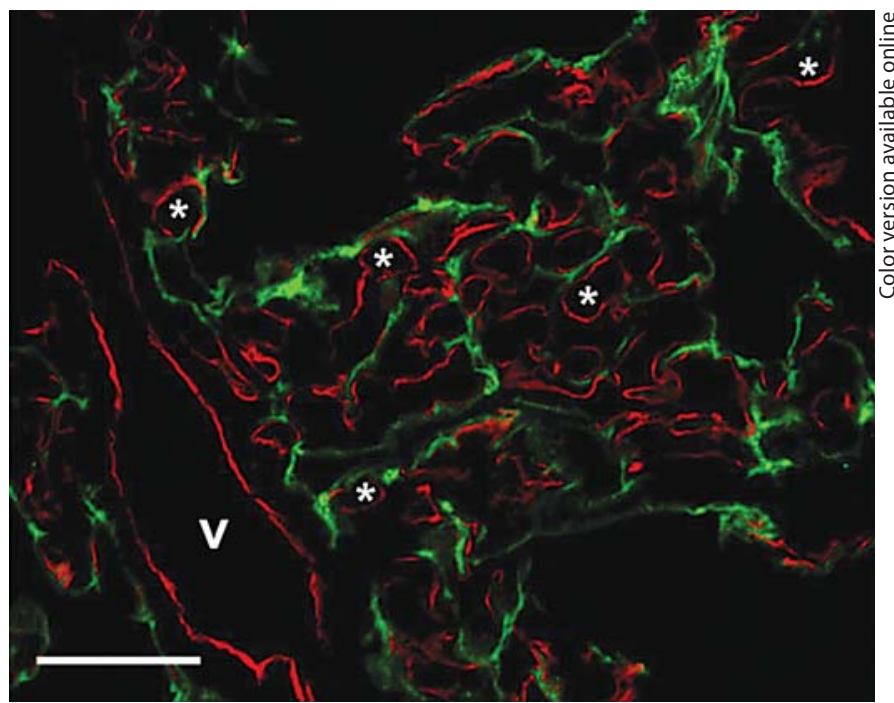

lymphocytes, nerves or the pleural mesothelium (not shown). Immunostaining was observed in all capillaries as well as all veins and veinules (fig. 1b, c), arteries and arterioles (not shown), of all sizes.

In addition to a dense vascular network, the lung also has an extensive lymphatic network. To determine whether lymphatic ECs were also immunostained, lung cryosections were analyzed by dual immunofluorescence 
with G278 and antipodoplanin, an antibody that specifically labels lymph vessels. Figure 2 shows that there is no colocalization of the G278 signal (in red) with the podoplanin signal (in green), indicating that G278 specifically labels the blood vascular endothelium.

\section{mAb G278 Recognizes podxl}

G278 does not effectively immunoprecipitate its cognate antigen isolated from $\mathrm{P}$, therefore in order to identify the protein target we used a gel-based liquid chromatography-tandem mass spectrometry approach (G-LC-MS/ MS), in which proteins in $\mathrm{P}$ are first resolved by SDSPAGE, then the appropriate band is excised, in-gel trypsin digested and the resulting peptides are analyzed by MS/ MS. The antigen recognized by G278 is solubilized by SDS, Triton X-100, NP40, CHAPS and $\beta$-octylglucoside (not shown). The latter detergent was selected for the GLC-MS/MS approach because, while it effectively solubilizes the target antigen, the majority of the proteins in $\mathrm{P}$ remain in the silica pellet following centrifugation, thus minimizing the presence of irrelevant proteins in the sample. G-LC-MS/MS analysis of the protein band corresponding to the Western blot signal identified the antigen as podxl. Eight unique peptides were identified, covering $14.4 \%$ of the rat podxl amino acid sequence (fig. $3 \mathrm{a}$, b). A typical MS/MS spectrum is shown in figure $3 \mathrm{c}$. In order to confirm the identity of the protein target for $\mathrm{mAb}$ G278, rat podxl was cloned and recombinantly expressed. Western blot analysis of the recombinant protein confirmed that mAb G278 specifically recognizes rat podxl (not shown) and moreover demonstrated that the epitope to which the antibody binds is resident in the protein core and not dependent on posttranslational modifications.

\section{Western Blotting and Proteomics Analysis of podxl}

Expression in Rat Heart, Kidney, Liver and Brain

Expression of podxl in other vascular beds was examined by Western blot analysis of homogenates and $\mathrm{P}$ isolated from rat brain, heart, liver, and kidney. As before, no signal was observed in homogenates prepared from lung, or in homogenates from brain, heart, or liver (fig. 4). A weak doublet was observed in the homogenate from kidney (fig. 4, lane 5), which is likely due to the presence of podxl in the podocytes of the glomeruli and in the thin segment of the loop of Henle (see below). podxl expression was observed in $\mathrm{P}$ isolated from each of the organs with the strongest signals being in lung and heart $\mathrm{P}$, followed by kidney, brain and liver P. podxl in $\mathrm{P}$ from heart and lung migrates as a doublet with a strong signal seen at approximately $130 \mathrm{kDa}$ and a weak-

\begin{tabular}{|c|c|c|}
\hline Position & Detected peptide sequence & $\begin{array}{c}\text { Charge } \\
\text { status }\end{array}$ \\
\hline $303-312$ & $\begin{array}{l}\text { FLELLCHSAK } \\
\end{array}$ & 2 \\
\hline 313-338 & ASFKPAEDSCALELAPILDNQAVAVK & 2 \\
\hline 313-338 & ASFKPAEDSCALELAPILDNQAVAVK & 3 \\
\hline $317-338$ & PAEDSCALELAPILDNQAVAVK & 2 \\
\hline 340-345 & IVIETK & 1 \\
\hline $357-374$ & DKWDDLTEAGVIDIHLGK & 3 \\
\hline 375-384 & EGPPEVNEDR & 1 \\
\hline 375-384 & EGPPEVNEDR & 2 \\
\hline
\end{tabular}

MRPTLALSALLLLQLLLLSTPSLSQDNGNKTDTSD ITSIDQNQDKPATNQPSNATPKSSVQPPTPTSIST SSPDPKATQSSNSSVTTTSDSTTDRTSSSTSTVPT TSNSGQTVSSGGKSSDKITTALPTTLGPVNASSQP TDLNTSTKLPSTPTTNSTASPHQPVSHSEGQHTTV QSSSASVSSSDNTTLLWILTTSKPTGTSEGTQPIA ISTPGITTPVSTPLQPTGSPGGTESVPTTEEFTHS TSSWTPVVSQGPSTPSSTWTSGSYKLKCDPAIKPH EELLILNLTRDSFCKGSPPNERFLELLCHSAKASF KPAED SCALELAPILDNQAVAVKRIVIETKLSPKA VFELLKDKWDDLTEAGVIDIHLGKEGPPEVNEDRF SLPLIITIVCMASFLLLVAALYGCCHQRISQRKDQ QRLTEELQTVENGYHDNPTLEVME TPSEMQEKKVV b NLNGELGDSWIVPLDNLTKEDLDEEEDTHL

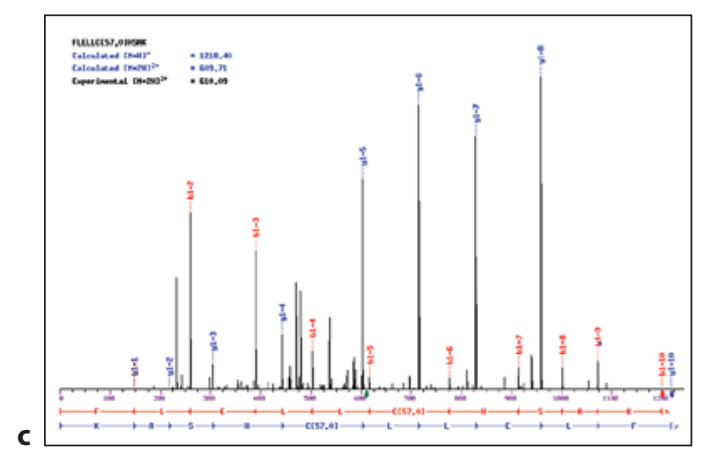

Fig. 3. G-LC-MS/MS analysis of the protein recognized by $\mathrm{mAb}$ G278. Proteins extracted by selective detergent solubilization were resolved by SDS-PAGE and the gel slice containing the target antigen was analyzed. a Unique proteolytic peptides of rat podxl (column 2) identified by G-LC-MS/MS. The primary amino acid positions are listed in column 1; the charge status of each identified peptide is listed in column 3. b Peptides (highlighted in blue) covering $14.4 \%$ of the rat podxl (NCBI: gi6492252) amino acid sequence which were identified in the excised gel band containing the target antigen. $\mathrm{c}$ A typical MS/MS spectrum, FLELLCHSAK.

er signal at approximately $120 \mathrm{kDa}$ (seen to better advantage in fig. 10c). In brain and kidney $\mathrm{P}$, only the 130 $\mathrm{kDa}$ band is observed while in liver $\mathrm{P}$ two bands of approximately 125 and $115 \mathrm{kDa}$ are seen. These differences are probably due to natural variations between organs since it is known that the molecular weight of rat podxl varies depending on the cell type [14]. The presence of podxl in the EC membranes from each of these organs was also confirmed by proteomics analyses. In 


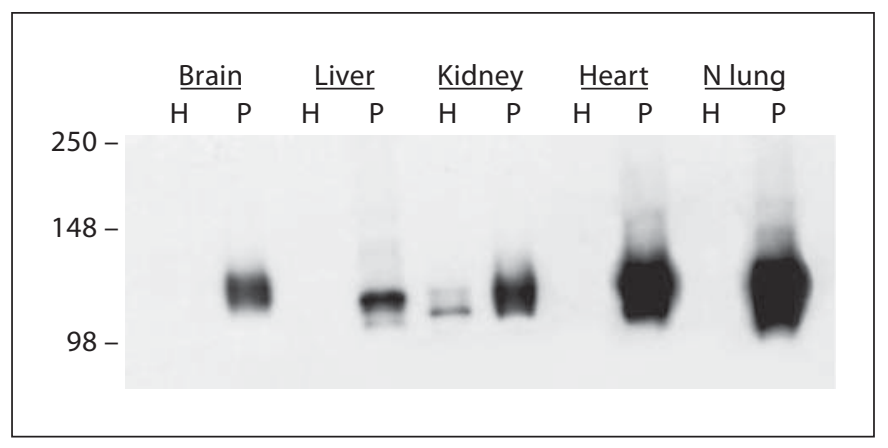

Fig. 4. Western blot analysis of podxl expression in rat organs. Homogenates $(\mathrm{H}, 5 \mu \mathrm{g})$ and $\mathrm{P}(5 \mu \mathrm{g})$ prepared from rat brain, liver, kidney, heart, and normal lung were analyzed by Western blotting with $\mathrm{mAb}$ G278. podxl is seen in P from all organs but is not detected in organ homogenates with the exception of kidney.

each case, we were able to detect at least one unique podxl peptide (not shown).

\section{Immunohistochemical Analysis of podxl Expression in} Other Rat Organs

The distribution of podxl in rat organs was examined by immunohistochemistry. G278 performed equally well on fresh-frozen, fixed-frozen and paraffin-embedded tissues, but immunostaining of paraffin-embedded sections is shown here because of the quality of the histological architechture. Analysis of the heart, kidney, liver and brain shows that the signals seen by Western blotting in figure 4 were from podxl expressed by the ECs. In these organs, as in lung tissue, all capillaries, arteries, arterioles, veins and veinules of all sizes were stained. In the heart,

Fig. 5. Immunohistochemical analysis of podxl expression in paraffin-embedded sections of normal rat organs. Blood vessels in all organs examined were clearly stained with mAb G278. a EC staining in heart; the endocardium is indicated by arrows. b Kidney cortex showing staining of peritubular vessels, capillaries and podocytes in the glomerulus (G) and a glomerular arteriole (A). c, d Kidney medulla and kidney papilla, respectively; vessels are indicated by arrowheads, thin segments of the loop of Henle are indicated by arrows. e Vessel staining in cerebrum; pia mater is indicated by arrows. $\mathbf{f}$ podxl expression of vessels in the cerebellum. g Portal area of liver showing staining of the portal vein (PV) and endothelia lining the sinusoids (arrows). $\mathrm{B}=$ Bile-collecting duct; $\mathrm{L}=$ lymphatic vessel. $\mathbf{h}$ Liver showing podxl expression in a hepatic veinule $(\mathrm{V})$ and in the endothelia lining surrounding sinusoids (arrows). i Panoramic view of skin; vessels throughout the thickness of the skin are immunostained with G278; the stain seen on hair follicles (arrows) is similar to that seen on control
G278 stained all blood vessels as well as the endocardium but showed no immunoreactivity with the cardiomyocytes (fig. 5a) or the epicardium (not shown). G278 also stained all blood vessels in the cortex, glomeruli, medulla and papilla of the kidney (fig. 5b-d). No extraendothelial stain was seen in the kidney with the exception of the podocytes in the glomeruli and the thin segment of the loop of Henle. Vessels in all parts of the brain examined also expressed podxl, but no staining was seen in the nervous tissue itself or the pia mater (fig. 5e, f). In the portal area of the liver, portal veins, hepatic arteries and other small vessels stained with G278, but no immunoreactivity was seen in the bile-collecting ducts, lymphatics or hepatocytes (fig. 5g). Hepatic venules and the endothelium lining the liver sinusoids also expressed podxl (fig. 5h), but the mesothelial covering was negative (not shown).

When the immunohistochemical analysis was extended to include skin, skeletal muscle, spleen, esophagus, stomach, colon, pancreas and bladder, we found that G278 also stained the endothelia in all of the blood vessels of these tissues as well. In the epidermis, dermis and hypodermis of the skin, ECs were the only cell type that stained with G278 (fig. 5i). Likewise, in skeletal muscle only the endothelium was stained (fig. 5j). podxl expression was also seen in the central and pulp arteries of the spleen (fig. 5k) as well as in the trabecular arteries and veins (not shown). Megakaryocytes, which are known to express podxl [14] were also stained (fig. 5k, inset) and only a few cells in the mesothelium on the surface of the spleen were immunopositive (not shown). In the esophagus, stomach and large intestine (fig. 5l-n), blood vessels in the muscularis mucosa and lamina propria of the mucosal layer, as

slides and is nonspecific. $\mathbf{j}$ Longitudinal section of skeletal muscle showing stained capillaries in the endomysium. $\mathbf{k}$ Spleen showing staining of central artery $\left(^{*}\right)$ in the white pulp (WP) and surrounding vessels in the red pulp (RP); inset shows a megakaryocyte stained with G278. I-n Panoramic views of esophagus, stomach and large intestine, respectively, showing vessel staining in the mucosa and lamina propria of the mucosal layer $(\mathrm{M})$, in the submucosa (S) and in the muscularis externa (ME). o Pancreas, showing staining of all vessels throughout the organ, and on the surface of the epithelium lining interlobular ducts (inset). I = Pancreatic islet; $\mathrm{D}=$ interlobular duct; $\mathrm{A}=$ interlobular arteriole. p Urinary bladder showing staining of vessels in the lamina propria (L) and muscularis (Ms), and staining of the mesothelium $\left.{ }^{*}\right)$; light staining was also seen on some portions of the transitional epithelium (arrows). Bar in $\mathbf{a}(\mathbf{b}-\mathbf{h})=20 \mu \mathrm{m}$; bar in $\mathbf{i}(\mathbf{j}-\mathbf{p})=$ $50 \mu \mathrm{m}$. 
well as those in the submucosa, and the muscularis externa were immunopositive. Blood vessels throughout the pancreas also expressed podxl (fig. 5o), including capillaries in the islets, as well as intralobular and interlobular vessels of all sizes. Staining was sometimes observed on the cells lining the interlobular ducts (fig. 5o, inset), but not on the epithelium of the intralobular ducts (not shown). In the bladder (fig. $5 p$ ), endothelial immunostaining was seen in blood vessels of the muscularis and the lamina propria. Staining was sometimes seen on the transitional epithelium lining the bladder, and a strong podxl signal was observed on the mesothelium.
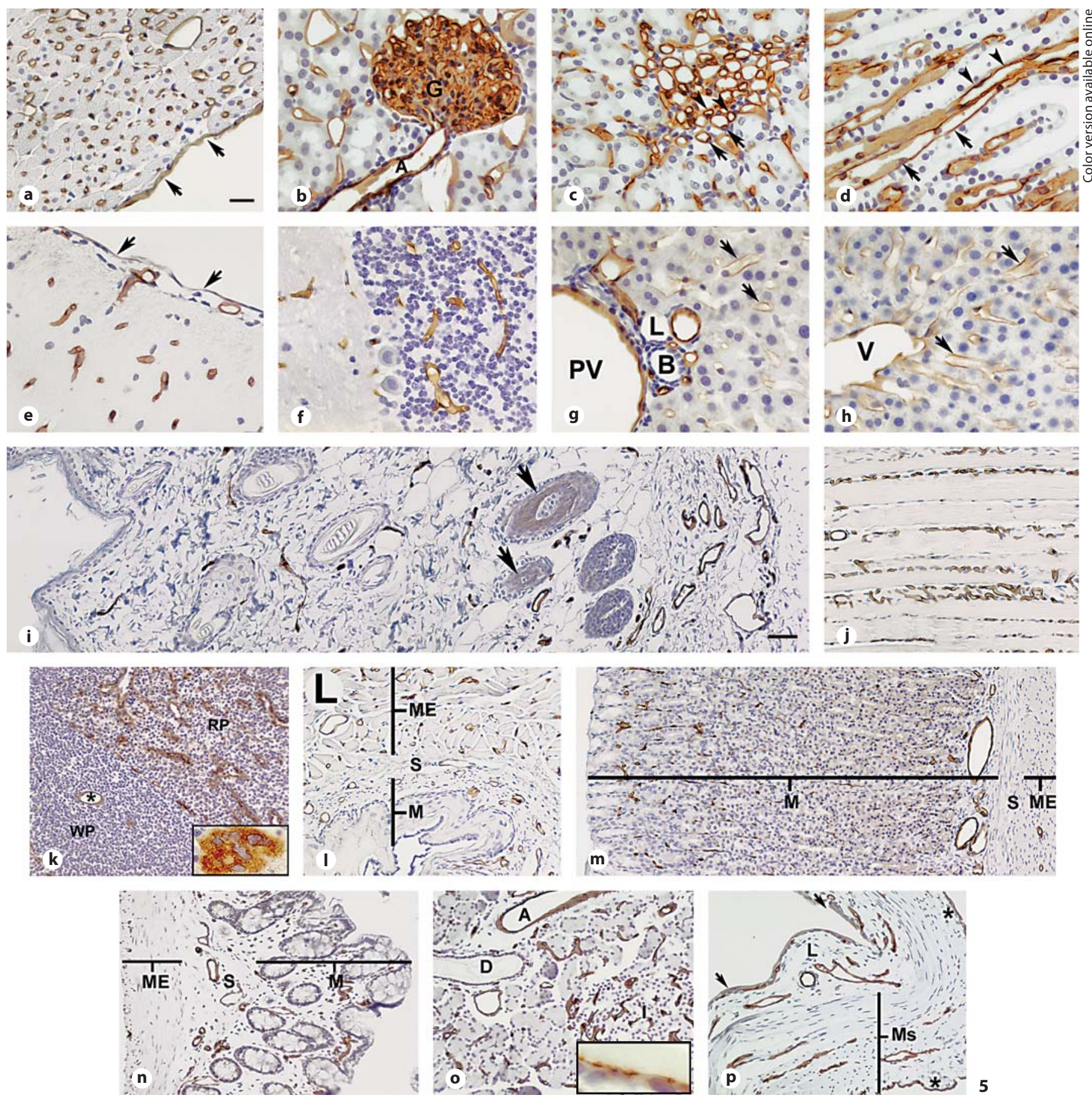

and Tumor Tissues 


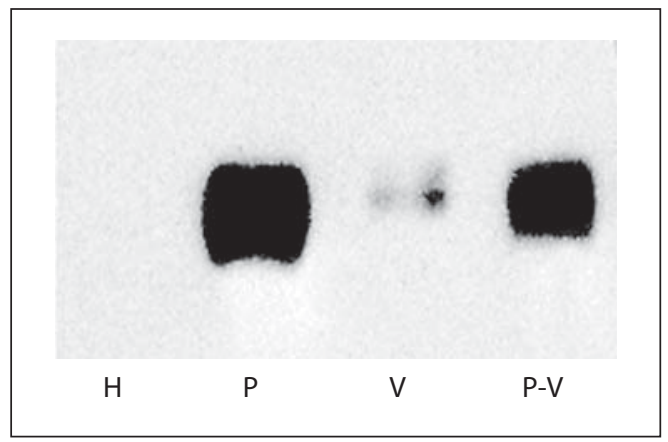

Fig. 6. podxl protein is not enriched in caveolae. Western blot of homogenate from normal lung $(\mathrm{H}, 5 \mu \mathrm{g})$, normal lung $\mathrm{P}(5 \mu \mathrm{g})$, caveolae (V, $2 \mu \mathrm{g})$, and the caveolae-depleted subfraction of $\mathrm{P}$ (P$\mathrm{V}, 5 \mu \mathrm{g})$ were probed with $\mathrm{mAb}$ G278. Strong signals are seen in $\mathrm{P}$ and in $\mathrm{P}-\mathrm{V}$, but very little is seen in $\mathrm{V}$, indicating that podxl is expressed primarily outside of the caveolae.

\section{podxl Is Not Expressed in Caveolae}

Continuous ECs are heavily invested with flask-shaped invaginations called caveolae which mediate the uptake and transport of blood-borne molecules across the endothelium $[13,15,16]$. Caveolae were isolated by further subfractionation of lung $\mathrm{P}$ and analyzed by Western blotting with G278. As shown in figure 6, strong podxl signals were seen in $\mathrm{P}$ and in $\mathrm{P}$ depleted of caveolae, but very little podxl could be detected in caveolae. When tissues were stained by dual immunofluorescence for caveolin-1 (the major protein in caveolae) and for podxl (fig. 7a) there was little or no colocalization of the signals when exact cross sections of capillaries (fig. $7 \mathrm{~b}$ ) or the endocardium (fig. 7c) were examined.

\section{In vivo Targeting}

Western blot analysis and immunohistochemistry showed that podxl is expressed in every vascular bed examined, but the accessibility of the antigen to binding by G278 via the blood stream could not be determined by these analyses. Planar gamma-scintigraphy was used on rats following tail vein injection of ${ }^{125} \mathrm{I}-\mathrm{G} 278$ in order to assess podxl targeting in various organs. Images were acquired at $10 \mathrm{~min}, 1,4,8$ and $24 \mathrm{~h}$ after injection. By 10 min, a clear, strong lung image was evident, and some accumulation could also be observed in kidney and liver (fig. 8a). At $1 \mathrm{~h}$, intensities of the lung and liver images had decreased slightly and continued to decline throughout the time course while at the same time the signal increased somewhat in kidney. Because podxl is not expressed in caveolae (fig. 6, 7) ${ }^{125} \mathrm{I}-\mathrm{G} 278$, once bound to the

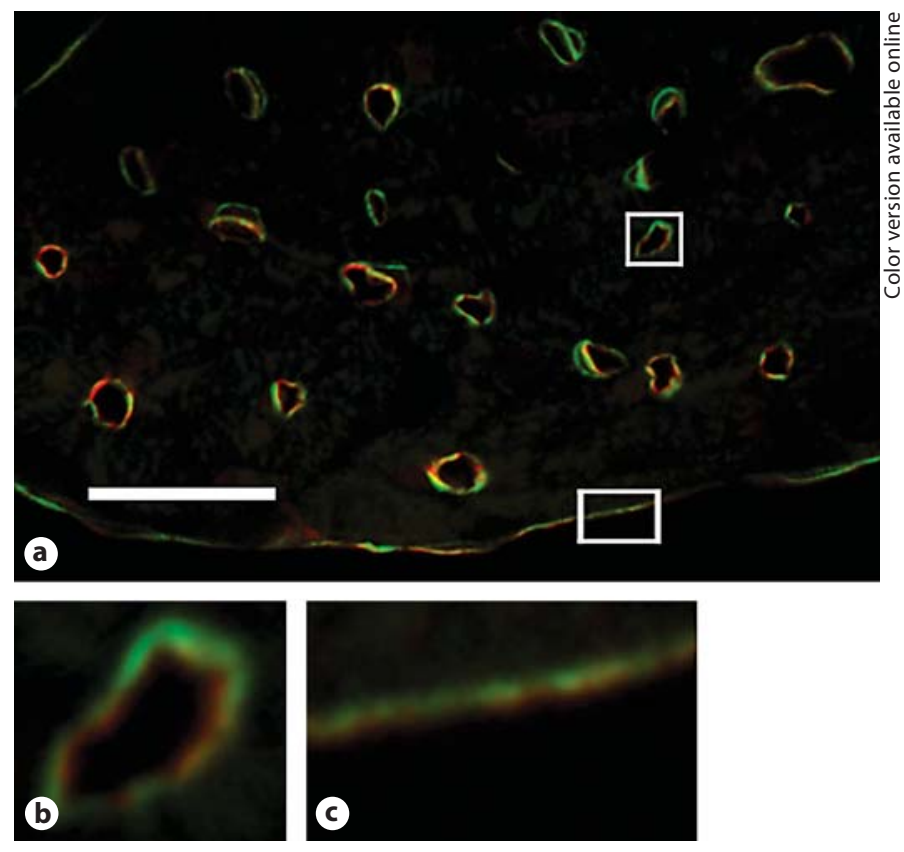

Fig. 7. Dual immunofluorescence analysis of rat heart sections immunostained with mAb G278 (red) and a goat pAb to the caveolar marker caveolin-1 (green). When seen in exact cross-section, there is little or no colocalization of the two signals in capillaries (square in $\mathbf{a}=\mathbf{b}$ ) nor in the endocardium (rectangle in $\mathbf{a}=$ c), indicating that podxl is primarily located outside of the caveolae. Scale bar in $\mathbf{a}=20 \mu \mathrm{m}$.

endothelium, likely would not be internalized but instead would remain on the luminal surface and be subjected to deiodination, which is known to occur in blood [17]. The redistribution of the signal from lung to kidney is likely due to the processes involved in eliminating free ${ }^{125} \mathrm{I}$. Consistent with this hypothesis is the appearance of a signal in thyroid seen at later time points (not shown). Clearer pictures of organ targeting were obtained with high-resolution pinhole single-photon-emission computed tomography (SPECT) imaging combined with Xray computed tomography $(\mathrm{CT})$. Figure $8 \mathrm{~b}-\mathrm{d}$ shows that $1 \mathrm{~h}$ after tail vein injection there is a very strong signal in lung and much weaker signals in the heart, liver and kidneys. When antibody binding was quantified in a biodistribution assay the results were consistent with the SPECT images. The majority of ${ }^{125} \mathrm{I}-\mathrm{G} 278$, slightly more than $30 \% \mathrm{ID} / \mathrm{g}$, was measured in lung $1 \mathrm{~h}$ after tail vein injection, while heart, liver, kidney and other organs bound substantially less (fig. 9a). Under the same conditions, little or no accumulation of a radiolabeled isotype-matched control antibody was detected in these tissues. At the 24- 

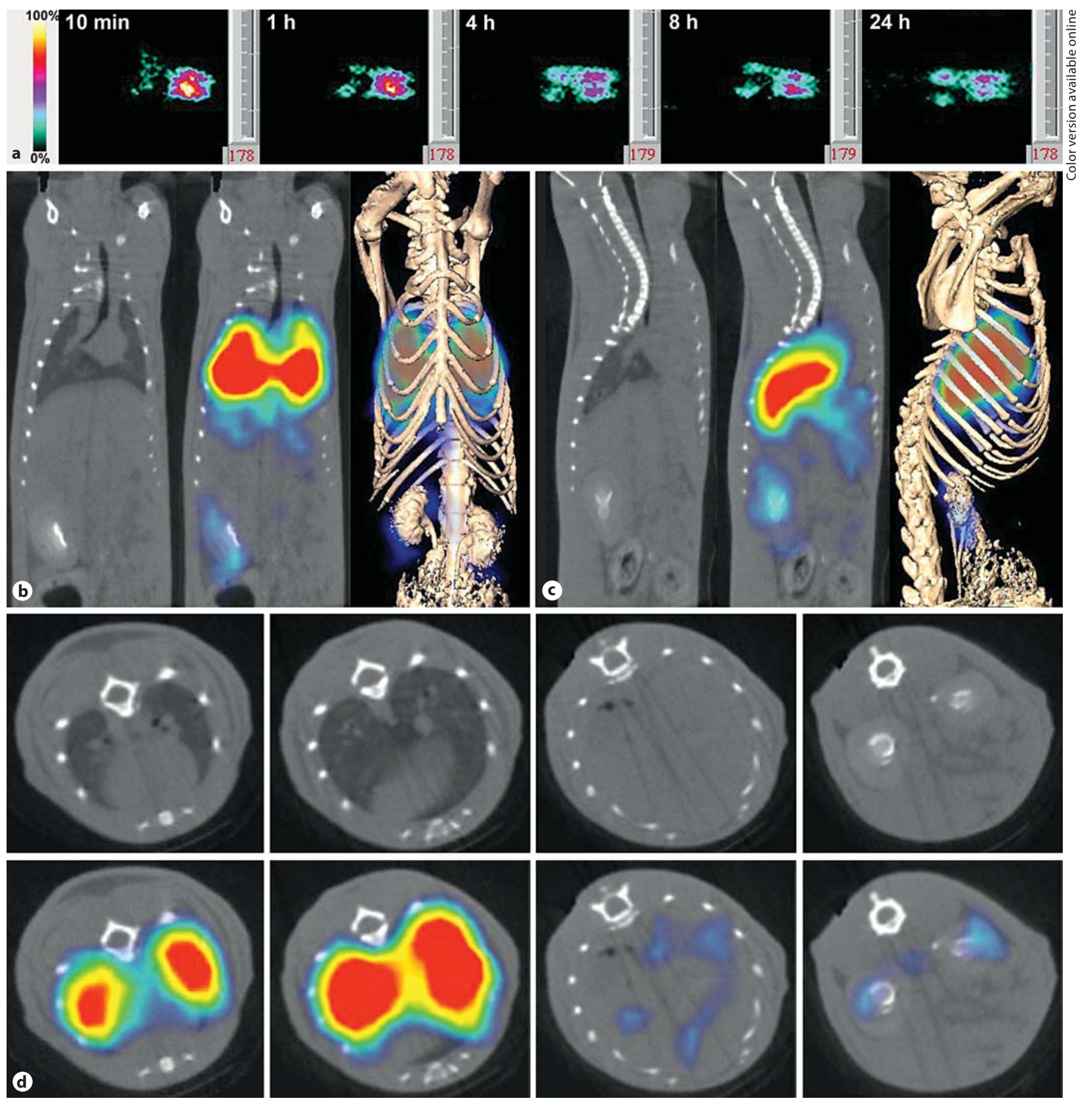

Fig. 8. In vivo imaging of organ immunotargeting. Rats were injected via the tail vein with ${ }^{125} \mathrm{I}-\mathrm{G} 278(10 \mu \mathrm{g}$ at $10 \mu \mathrm{Ci} / \mu \mathrm{g})$. a Planar gamma-scintigraphic images were acquired at $10 \mathrm{~min}$ and at $1,4,8$, and $24 \mathrm{~h}$. At $10 \mathrm{~min}$, the antibody has accumulated primarily in the lungs, but signals can also be seen in the areas of the liver and kidneys. b-d SPECT/CT imaging of G278 targeting showing coronal (b), saggital (c) and axial slices (d). The far right

images in $\mathbf{b}, \mathbf{c}$ show the fusion of volumetric SPECT texture with the CT isosurface. Axial slices in $\mathbf{d}$ were taken at the level of the heart and lungs (far left), the lungs (second from left), the liver (third from left) and the kidneys (far right). Because of the high signal in lungs, the threshold is too low to see bound radiolabel in the heart. 


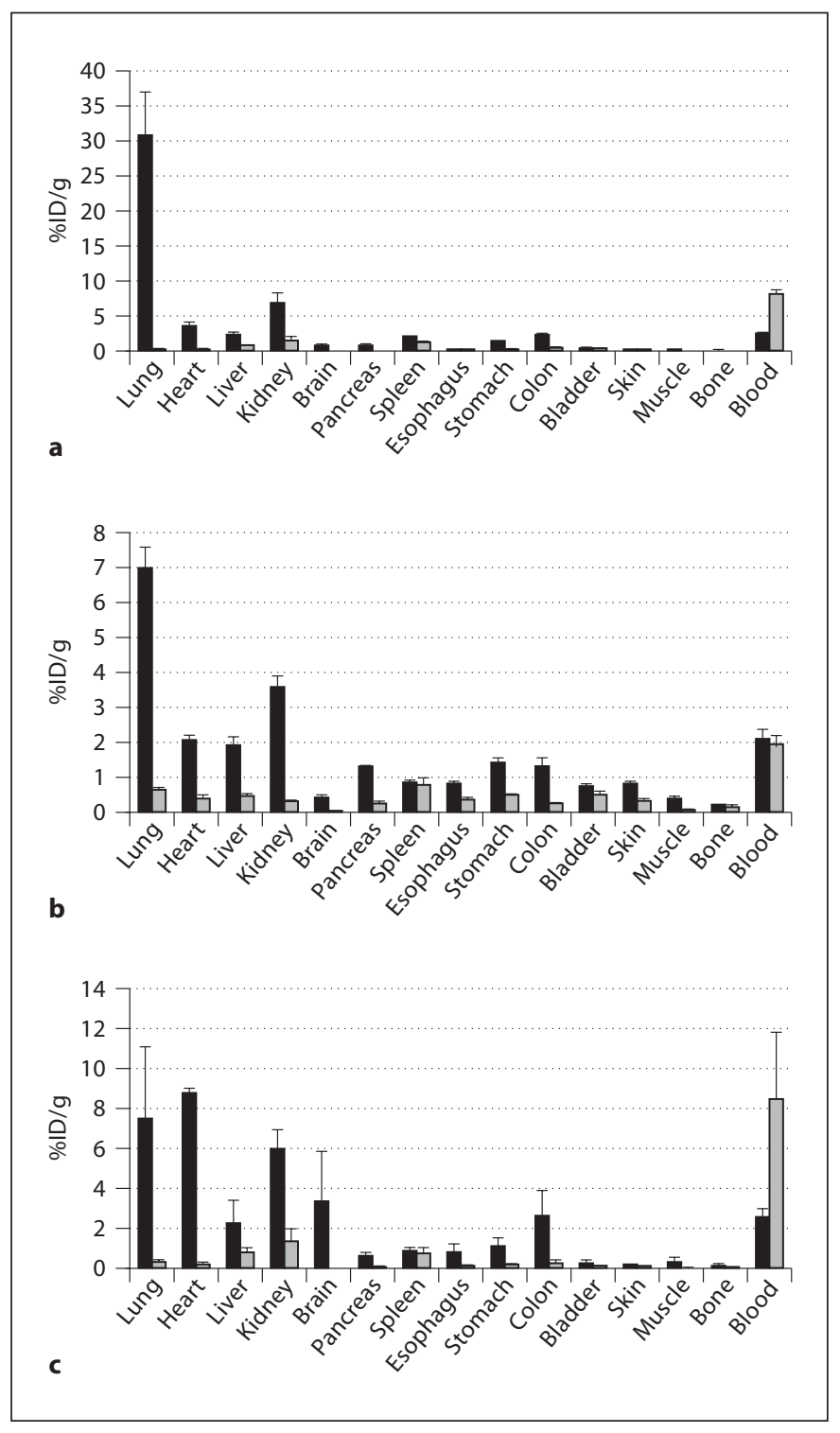

Fig. 9. Biodistribution analysis of G278. ${ }^{125} \mathrm{I}-\mathrm{G} 278$ (black bars) or a radiolabeled isotype-matched control antibody (gray bars) was injected into rats via the tail vein $(\mathbf{a}, \mathbf{b})$ or via the left ventricle (c). Tissues and blood samples were harvested at $1 \mathrm{~h} \mathrm{(a,} \mathrm{c)} \mathrm{or} 24 \mathrm{~h} \mathrm{(b)}$, weighed and gamma-radioactivity was measured. Results are expressed as percent injected dose per gram of tissue (\%ID/g).

hour time point, there was roughly a four-fold decrease in ${ }^{125}$ I-G278 found in the lung (fig. 9b). There was also a decrease in radioactivity in the heart, liver and kidney, but the magnitude was not as great as that seen in the lung. In these experiments, the measured radioactivity in the various organs was inconsistent with the amounts of podxl seen in Western blots of lung, heart, kidney, liver and brain (fig. 4). This may be due to the fact that when reagents are inoculated via the tail vein, the first capillary bed that will be encountered is in the lungs, where a large portion of the injected molecule could be removed by this highly vascularized organ. In order to allow G278 to pass once through the vasculature of other organs in the body prior to entering the lung, the radiolabeled G278 or an isotype-matched control was injected via the left ventricle and radioactivity was measured at the 1-hour time point as before. As seen in figure $9 c$, bound ${ }^{125} \mathrm{I}-\mathrm{G} 278$ in most organs was similar to that seen in animals injected by tail vein and there was a slight decrease (15\%) in kidney. However, bound ${ }^{125} \mathrm{I}-\mathrm{G} 278$ increased 4.6 -fold in brain and 2.5-fold in heart, indicating that the podxl expressed in these vascular beds is accessible and will bind G278. The majority of the isotype-matched control antibody remained in circulation and there was little or no accumulation of this antibody in the other tissues.

\section{podxl Expression Can Be Modulated in the Tumor Microenvironment}

Tumor blood vessels differ from their normal counterparts in a number of parameters, including size, shape and permeablilty. Using a subtractive proteomics approach, we have previously identified alterations in the protein profiles of endothelia isolated from normal rat lungs and from tumor-bearing lungs [5]. We wished to determine whether podxl expression was affected by the tumor microenvironment using the same 13762 rat mammary carcinoma model as well as the FRcJ-4 rat sarcoma model [18]. When sections of lungs bearing FRcJ-4 nodules were immunostained with G278, podxl expression was seen on vessels in the normal portions of the lung and on the endothelium throughout the tumor mass itself (fig. 10a). Likewise, examination of 13762-tumor lungs showed podxl expression on vessels in the normal lung tissue and in the outer margins of the tumor nodule. However, in the deeper portions of the tumor, podxl expression was diminished or absent (fig. 10b). Microscopic examination of these deeper vessel-like structures showed that they were composed of cells which were similar to and continuous with podxl-positive ECs (fig. 10b, arrowheads). Western blot analysis of $\mathrm{P}$ isolated from normal and 13762-tumor-bearing lungs showed that podxl expression was comparable in the two samples (fig. 10c). These data indicate that podxl expression in the normal areas of tumor-bearing lungs is not affected by the presence of the tumors and that the tumor microenvironment can influence podxl expression, but varies from tumor to tumor. Loss of an EC marker in the tumor microenvironment was not limited to podxl. Figure 10d 


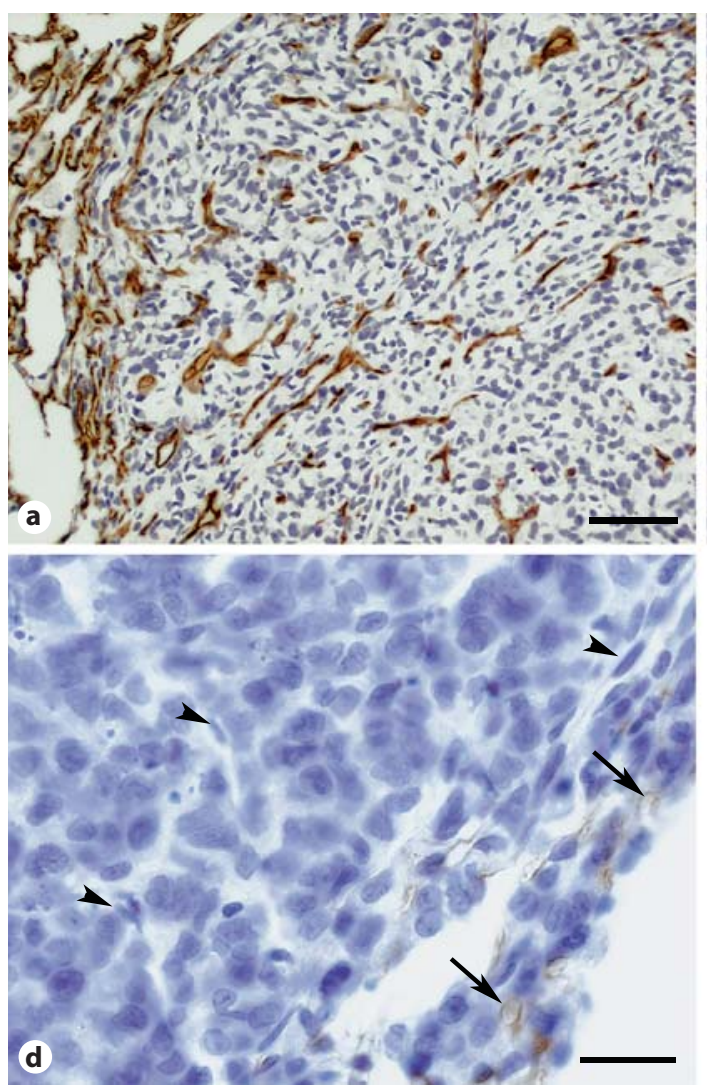

Fig. 10. Analysis of podxl expression in rat metastatic mammary tumor and sarcoma models. a Metastatic lung tumors of the rat sarcoma cell line FRcJ-4 were immunostained with G278. Signals are observed in the adjacent normal lung tissue and throughout the tumor mass itself. b Metastatic lung tumors of the rat mammary carcinoma cell line 13762 were immunostained with G278. podxl expression is observed in the vessels adjacent to the tumor and in the corona of the tumor itself. Deeper in the tumor nodule, podxl expression by ECs is diminished (arrows) or lost (arrowheads). c Homogenates $(\mathrm{H})$ and $\mathrm{P}$ isolated from normal rat lungs (N lung) and tumor-bearing lungs ( $\mathrm{T}$ lung) analyzed by Western blots probed with mAb G278. Molecular weight markers are shown on the left. d, e Anti-PECAM/CD31 immunostaining of tumor and normal vessels. PECAM/CD31 expression is seen in vessels in the corona of a 13762-tumor and adjacent normal lung (d, e, arrows), but is absent in ECs found deeper in the tumor mass (d, arrowheads). f Dual immunofluorescence of a section cut through the corona of a 13762 mammary carcinoma nodule. There is no colocalization of the G278 signal (red channel) and anti-podoplanin (green channel), showing that mAb G278 does not immunostain the tumor lymphatic endothelium. Bar in a, $\mathbf{b}=50 \mu \mathrm{m}$; bar in $\mathbf{d}, \mathbf{f}=20 \mu \mathrm{m}$. shows that PECAM/CD31 was detectable in blood vessels at the perimeter of the tumor and in the adjacent normal lung tissue (fig. 10e) but was absent from vessel-like structures within the tumor mass (fig. 10d). We also used dual immunofluorescence to evaluate the ability of $\mathrm{mAb}$ G278 to distinguish blood vascular ECs from lymphatic ECs within the tumor. Figure $10 \mathrm{f}$ shows a section through the outer margin of a tumor nodule in which there is no colocalization of mAb G278 (red) with antipodoplanin (green), indicating that tumor lymphatics, like those in normal tissue (see fig. 2), are not recognized by $\mathrm{mAb}$ G278.

Rat Podocalyxin Expression in Normal and Tumor Tissues

\section{Discussion}

podxl, along with CD34 and endoglycan, belongs to the CD34 family of sialomucins, which are similar in structure and genomic organization [for a review, see 19]. In the present study, we examined the distribution of podxl on rat endothelia in normal and tumor tissues using $\mathrm{mAb}$ G278, developed in our laboratory. G278 binds to the protein core of this heavily sialylated and O-glycosylated member of the sialomucin protein family and, as such, it is likely able to detect all forms of rat podxl irrespective of the nature and extent of posttranslational 
modification. This feature was exploited in the present study to determine the expression levels of podxl in different vascular beds. Western blot analysis of luminal plasma membranes isolated from rat lung, heart, brain, liver and kidney showed that podxl is most highly expressed on the ECs in lung and heart. The epitope recognized by G278 is exposed on the luminal EC surface, which allowed us to image various organs and to determine the biodistribution of radiolabled antibody delivered into the blood stream. When inoculated via the tail vein, the antibody was delivered to the 'first-pass' organ, namely the lungs, and bound primarily in that vascular bed. However, delivery via the left ventricle allowed the antibody to reach other organs prior to entering the lungs, which altered the biodistribution profile of ${ }^{125} \mathrm{I}-\mathrm{G} 278$. Using both multiorgan Western blot analysis and biodistribution, we have shown that the podxl protein is most highly expressed in rat lung and heart.

podxl was first identified as the major sialoprotein of glomerular podocytes [20] and was subsequently shown to be expressed on rat endothelia by indirect immunofluorescence [20-22]. We have expanded on the latter studies and have examined podxl expression in a number of rat organs by immunohistochemistry. G278 is capable of immunostaining fresh-frozen and fixed-frozen tissues as well as formalin-fixed paraffin-embedded tissues. Formalin-fixed paraffin-embedded sections offer the advantage of preserving the histological architecture of the organ, allowing the investigator to better appreciate the relationship of stained cells with surrounding cells and stroma. Indeed, using dual immunofluorescence, we were able to resolve the difference in location of podxl on the luminal EC plasma membrane and caveolin-1 on the cytoplasmic face of the membrane. Western blotting of caveolae isolated from rat lung $\mathrm{P}$ also demonstrated that little or no podxl is found in these organelles. These results are consistent with the immuno-electron microscopy of Horvat et al. [21], which showed no vesicle staining in lung ECs with a rabbit pAb.

Lin et al. [23] demonstrated that podxl is universally expressed by ECs in the dog. These authors, however, also reported podxl expression in a number of extravascular locations (heart muscle fibers, skeletal muscle, neuronal cells in the cerebrum) which were not observed in G278stained rat tissues. These differences may reflect variations in podxl distribution in the two species. The only extraendothelial staining that we observed in rat tissues was in cells known to express podxl, namely the podocytes $[20,22,24]$, the thin segment of the loop of Henle and mesothelium [23], and megakaryocytes [14]. In addi- tion, we found occasional staining on the epithelial cells lining the interlobular ducts of the pancreas and on some transitional epithelial cells in the bladder. The presence of podxl in pancreatic ductal epithelial cells is of interest in light of the report by Ney et al. [25] that identified podxl as useful marker to differentiate pancreatic ductal adenocarcinomas from other tumor types. They found that $44 \%$ of the ductal tumors expressed podxl. Our observation that podxl is expressed on some epithelial cells in the interlobular but not the intralobular ducts raises the question as to whether this protein might serve as a suitable marker to further define the origins of subsets of pancreatic ductal adenocarcinomas. Lin et al. [23] did not report on podxl expression in lymph vessels of the dog, but in the present study we found that in the rat $\mathrm{mAb}$ G278 did not cross-react with lymphatic ECs. Our results show that G278 can clearly discriminate between blood and lymphatic ECs in both normal and tumor tissues.

mAb G278 works exceptionally well to immunostain rat blood vessels of all types and all sizes and consistently outperformed antibodies to the more commonly used pan-endothelial markers CD34 [Testa et al., submitted] and PECAM/CD31 (fig. 10d, e). Commercially available anti-PECAM pAbs which cross-react with rat PECAM are produced by immunization with the human protein, which shares only a $60 \%$ identity with the rat homolog. Poor immunostaining with these antibodies may be the result of less than optimal reactivity with rat PECAM. In addition, brief ( $5 \mathrm{~min}$ ) formalin fixation of cryosections eliminated reactivity of the commercial anti-PECAM antibodies with rat ECs but did not affect immunostaining of human ECs (not shown). Formalin fixation did not eliminate rat EC immunostaining by $\mathrm{mAb}$ G278. We have also compared mAb G278 and anti-PECAM pAbs with respect to immunoisolation of rat ECs and found that $\mathrm{mAb}$ G278 was far superior in this function as well.

In this study, we have demonstrated that podxl is expressed on the normal vasculature of adult rat organs as well as in the tumor microenvironment where angiogenic processes are ongoing. This latter observation is consistent with previous reports of podxl expression in embryonic and extraembryonic vessels during development $[26,27]$. Interestingly, we have observed that rat ECs will lose podxl expression with continued in vitro culture [3]. The only other situation in which we have seen an absence of podxl is in the apparent vasculature in the interior of metastatic mammary tumor nodules growing in lung. The inner vessels in these tumors also lacked PECAM/CD31 expression. Heukamp et al. [28] also observed that podxl and another EC marker, CD34, were 
absent from the microvasculature of dysplastic hepatic nodules. Modulation of podxl and other EC proteins in the tumor vasculature is not surprising. Studies from our laboratory [5] and others [29-32], using a variety of techniques, have compared normal and tumor ECs in an effort to identify tumor vascular markers. These reports have demonstrated a profound effect on the protein profiles of ECs within the tumor microenvironment. Although podxl was absent in the deeper portions of 13762 tumors, the loss was not detectable by Western blot analysis of $\mathrm{P}$ from normal and tumor lung. This may be due to a slight upregulation of podxl expression in the tumor margin and/or the adjacent normal tissue which would not have been evident by immunohistochemistry. Also of note was the fact that the molecular weights of podxl in tumor and normal lung $\mathrm{P}$ were the same. The molecular weight of rat podxl can vary depending on the cellular source [see for example 14], and in human tumor cells the molecular weight of podxl can be substantially larger than in normal tissue [33]. The observed differences in molecular weights are likely due to alterations in the extensive posttranslational modifications that constitute the majority of the protein's mass. In the present study, we observed no difference in the molecular weights of the podxl bands in $\mathrm{P}$ from normal or tumor lung. Although influenced by the tumor microenvironment, tumor ECs are not transformed cells and retain many features of normal ECs. Thus modification of podxl in tumor and normal ECs would be similar, resulting in proteins of the same molecular weights. The mechanism(s) which underlie the loss of podxl expression is unknown, and although its implications have not been elucidated, we have observed that in 13762 tumors where podxl expression is lost, the core of the nodule is almost always necrotic, while in the FRcJ-4 tumors necrotic centers were not of- ten seen. It has been suggested that the charge repulsion effects supported by this strongly anionic molecule may help to maintain open vascular lumens and glomerular filtration slits [14, 34]; however, the cause-and-effect relationship between podxl expression and tumor vessel patency remains to be determined.

podxl has been assigned both adhesive and anti-adhesive functions. In podxl knockout mice, the podocytes form tight and adherens junctions rather than the normal foot processes and slit diaphragms, and the pups die within $24 \mathrm{~h}$ of birth with anuria [35]. Studies have also shown that podxl acts as a ligand for L-selectin on high endothelial venules [36] and enhances cell adhesion to platelets [37]. Conversely, several transfection studies have suggested that podxl acts to disrupt the formation of cell-tocell junctions $[38,39]$ and leads to increased tumor cell migration and invasion in vitro [40]. podxl expression has also been associated with more aggressive forms of prostate [41] and breast cancer [38] and is a commonly expressed marker of blasts in acute leukemia [42].

$\mathrm{mAb}$ G278 is a robust antibody that reliably binds to podxl in a variety of biochemical, immunological and in vivo applications. We believe G278 to be an invaluable tool not only as a means of immunostaining rat blood vessels, but as reagent with which to elucidate the functions of podxl.

\section{Acknowledgements}

This work was supported by grants (to J.E.S.) from the National Cancer Institute (P01CA104898, R01CA83989 and R24CA095893) and National Heart Lung and Blood Institute (R01-HL074063, R01-HL052766 and R01-HL058216). We thank Kally Pascua, Alexina Wempren, Melinda Schnitzer and Michelle Pacheco for their excellent technical assistance.

\section{References}

1 Schnitzer JE: Update on the cellular and molecular basis of capillary permeability. Trends Cardiovasc Med 1993;3:124-130.

2 Carmeliet P: Angiogenesis in life, disease and medicine. Nature 2005;438:932-936.

- 3 Durr E, Yu J, Krasinska KM, Carver LA, Yates JR, Testa JE, Oh P, Schnitzer JE: Direct proteomic mapping of the lung microvascular endothelial cell surface in vivo and in cell culture. Nat Biotechnol 2004;22:985992.

\footnotetext{
4 McIntosh DP, Tan XY, Oh P, Schnitzer JE: Targeting endothelium and its dynamic caveolae for tissue-specific transcytosis in vivo: a pathway to overcome cell barriers to drug and gene delivery. Proc Natl Acad Sci USA 2002;99:1996-2001.

5 Oh P, Li Y, Yu J, Durr E, Krasinska KM, Carver LA, Testa JE, Schnitzer JE: Subtractive proteomic mapping of the endothelial surface in lung and solid tumours for tissue-specific therapy. Nature 2004;429:629635.
}

\footnotetext{
6 Valadon P, Garnett JD, Testa JE, Bauerle M, Oh P, Schnitzer JE: Screening phage display libraries for organ-specific vascular immunotargeting in vivo. Proc Natl Acad Sci USA 2006; 103:407-412.

7 Oh P, Schnitzer JE: Immunoisolation and subfractionation of plasma membranes to purify caveolae separately from glycosylphosphatidylinositol-anchored protein microdomains; in Celis J (ed): Cell Biology: A Laboratory Handbook. New York, Academic Press, 1998, pp 34-46.
} 
-8 Schnitzer JE, McIntosh DP, Dvorak AM, Liu J, Oh P: Separation of caveolae from associated microdomains of GPI-anchored proteins. Science 1995;269:1435-1439.

$\checkmark 9$ Rizzo V, Morton C, DePaola N, Schnitzer JE, Davies PF: Recruitment of endothelial caveolae into mechanotransduction pathways by flow conditioning in vitro. Am J Physiol Heart Circ Physiol 2003;285:H1720-H1729.

-10 Schnitzer JE, Liu J, Oh P: Endothelial caveolae have the molecular transport machinery for vesicle budding, docking, and fusion including VAMP, NSF, SNAP, annexins, and GTPases. J Biol Chem 1995;270:1439914404.

-11 Schnitzer JE, Oh P: Aquaporin-1 in plasma membrane and caveolae provides mercurysensitive water channels across lung endothelium. Am J Physiol 1996;270:H416H422.

12 Harlow E, Lane D: Antibodies: A Laboratory Manual. Cold Spring Harbor, Cold Spring Harbor Laboratory Press, 1988.

-13 Oh P, Borgstrom P, Witkiewicz H, Li Y, Borgstrom BJ, Chrastina A, Iwata K, Zinn KR, Baldwin R, Testa JE, Schnitzer JE: Live dynamic imaging of caveolae pumping targeted antibody rapidly and specifically across endothelium in the lung. Nat Biotechnol 2007;25:327-337.

14 Miettinen A, Solin ML, Reivinen J, Juvonen E, Vaisanen R, Holthofer H: Podocalyxin in rat platelets and megakaryocytes. Am J Pathol 1999;154:813-822.

-15 Oh P, McIntosh DP, Schnitzer JE: Dynamin at the neck of caveolae mediates their budding to form transport vesicles by GTP-driven fission from the plasma membrane of endothelium. J Cell Biol 1998;141:101-114.

-16 Schnitzer JE, Oh P, McIntosh DP: Role of GTP hydrolysis in fission of caveolae directly from plasma membranes. Science 1996; 274:239-242.

- 17 Russell J, O’Donoghue JA, Finn R, Koziorowski J, Ruan S, Humm JL, Ling CC: Iodination of annexin $\mathrm{v}$ for imaging apoptosis. J Nucl Med 2002;43:671-677.

-18 Kraemer M, Tournaire R, Dejong V, Montreau N, Briane D, Derbin C, Binetruy B: Rat embryo fibroblasts transformed by c-Jun display highly metastatic and angiogenic activities in vivo and deregulate gene expression of both angiogenic and antiangiogenic factors. Cell Growth Differ 1999;10:193200.

19 Furness SG, McNagny K: Beyond mere markers: Functions for CD34 family of sialomucins in hematopoiesis. Immunol Res 2006;34:13-32.
20 Kerjaschki D, Sharkey DJ, Farquhar MG: Identification and characterization of podocalyxin - the major sialoprotein of the renal glomerular epithelial cell. J Cell Biol 1984;98: 1591-1596.

21 Horvat R, Hovorka A, Dekan G, Poczewski H, Kerjaschki D: Endothelial cell membranes contain podocalyxin - the major sialoprotein of visceral glomerular epithelial cells. J Cell Biol 1986;102:484-491.

22 Miettinen A, Dekan G, Farquhar MG: Monoclonal antibodies against membrane proteins of the rat glomerulus. Immunochemical specificity and immunofluorescence distribution of the antigens. Am J Patho 1990;137:929-944.

23 Lin WL, Pang VF, Liu CH, Chen JY, Shen KF, Lin YY, Yu CY, Hsu YH, Jou TS: Pleomorphic extra-renal manifestation of the glomerular podocyte marker podocalyxin in tissues of normal beagle dogs. Histochem Cell Biol 2007;127:399-414.

24 Dekan G, Miettinen A, Schnabel E, Farquhar MG: Binding of monoclonal antibodies to glomerular endothelium, slit membranes, and epithelium after in vivo injection. Localization of antigens and bound IGGs by immunoelectron microscopy. Am J Pathol 1990;137:913-927.

-25 Ney JT, Zhou H, Sipos B, Buttner R, Chen X, Kloppel G, Gutgemann I: Podocalyxin-like protein 1 expression is useful to differentiate pancreatic ductal adenocarcinomas from adenocarcinomas of the biliary and gastrointestinal tracts. Hum Pathol 2007;38:359364.

26 Doyonnas R, Nielsen JS, Chelliah S, Drew E, Hara T, Miyajima A, McNagny KM: Podocalyxin is a CD34-related marker of murine hematopoietic stem cells and embryonic erythroid cells. Blood 2005; 105:4170-4178.

-27 McNagny KM, Pettersson I, Rossi F, Flamme I, Shevchenko A, Mann M, Graf T: Thrombomucin, a novel cell surface protein that defines thrombocytes and multipotent hematopoietic progenitors. J Cell Biol 1997;138: 1395-1407.

28 Heukamp LC, Fischer HP, Schirmacher P, Chen X, Breuhahn K, Nicolay C, Buttner R, Gutgemann I: Podocalyxin-like protein 1 expression in primary hepatic tumours and tumour-like lesions. Histopathology 2006; 49:242-247.

29 Herbert JM, Stekel D, Sanderson S, Heath VL, Bicknell R: A novel method of differential gene expression analysis using multiple cDNA libraries applied to the identification of tumour endothelial genes. BMC Genomics 2008;9:153.

30 Ran S, Downes A, Thorpe PE: Increased exposure of anionic phospholipids on the surface of tumor blood vessels. Cancer research 2002;62:6132-6140.
31 Rybak JN, Roesli C, Kaspar M, Villa A, Neri D: The extra-domain a of fibronectin is a vascular marker of solid tumors and metastases. Cancer Res 2007;67:10948-10957.

-32 St Croix B, Rago C, Velculescu V, Traverso G, Romans KE, Montgomery E, Lal A, Riggins GJ, Lengauer C, Vogelstein B, Kinzler KW: Genes expressed in human tumor endothelium. Science 2000;289:1197-1202.

>33 Schopperle WM, Kershaw DB, DeWolf WC: Human embryonal carcinoma tumor antigen, GP200/GCTM-2, is podocalyxin. Biochem Biophys Res Commun 2003;300:285290.

34 Dekan G, Gabel C, Farquhar MG: Sulfate contributes to the negative charge of podocalyxin, the major sialoglycoprotein of the glomerular filtration slits. Proc Natl Acad Sci USA 1991;88:5398-5402.

-35 Doyonnas R, Kershaw DB, Duhme C, Merkens H, Chelliah S, Graf T, McNagny KM: Anuria, omphalocele, and perinatal lethality in mice lacking the CD34-related protein podocalyxin. J Exp Med 2001;194: 13-27.

-36 Sassetti C, Tangemann K, Singer MS, Kershaw DB, Rosen SD: Identification of podocalyxin-like protein as a high endothelial venule ligand for L-selectin: Parallels to CD34. J Exp Med 1998;187:1965-1975.

37 Larrucea S, Butta N, Rodriguez RB, AlonsoMartin S, Arias-Salgado EG, Ayuso MS, Parrilla R: Podocalyxin enhances the adherence of cells to platelets. Cell Mol Life Sci 2007;64:2965-2974.

-38 Somasiri A, Nielsen JS, Makretsov N, McCoy ML, Prentice L, Gilks CB, Chia SK, Gelmon KA, Kershaw DB, Huntsman DG, McNagny KM, Roskelley CD: Overexpression of the anti-adhesin podocalyxin is an independent predictor of breast cancer progression. Cancer Res 2004;64:5068-5073.

$>39$ Takeda T, Go WY, Orlando RA, Farquhar MG: Expression of podocalyxin inhibits cell-cell adhesion and modifies junctional properties in Madin-Darby canine kidney cells. Mol Biology Cell 2000;11:3219-3232.

40 Sizemore S, Cicek M, Sizemore N, Ng KP, Casey G: Podocalyxin increases the aggressive phenotype of breast and prostate cancer cells in vitro through its interaction with ezrin. Cancer Res 2007;67:6183-6191.

-41 Casey G, Neville PJ, Liu X, Plummer SJ, Cicek MS, Krumroy LM, Curran AP, McGreevy MR, Catalona WJ, Klein EA, Witte JS: Podocalyxin variants and risk of prostate cancer and tumor aggressiveness. Hum Mol Genet 2006;15:735-741.

42 Kelley TW, Huntsman D, McNagny KM, Roskelley CD, Hsi ED: Podocalyxin: a marker of blasts in acute leukemia. Am J Clin Pathol 2005;124:134-142. 